BMJ Open

Diabetes

Research

\& Care

\section{Histamine causes an imbalance between pro-angiogenic and anti-angiogenic factors in the retinal pigment epithelium of diabetic retina via $\mathrm{H} 4$ receptor/p38 MAPK axis}

To cite: Lee BJ, Byeon HE, Cho CS, et al. Histamine causes an imbalance between proangiogenic and anti-angiogenic factors in the retinal pigment epithelium of diabetic retina via $\mathrm{H} 4$ receptor/p38 MAPK axis. BMJ Open Diab Res Care 2020;8:e001710. doi:10.1136/ bmjdrc-2020-001710

- Supplemental material is published online only. To view, please visit the journal online (http://dx.doi.org/10.1136/ bmjdrc-2020-001710).

BJL and HEB contributed equally.

Received 20 June 2020 Revised 21 October 2020 Accepted 29 0ctober 2020

Check for updates

(c) Author(s) (or their employer(s)) 2020. Re-use permitted under CC BY-NC. No commercial re-use. See rights and permissions. Published by BMJ.

For numbered affiliations see end of article.

Correspondence to Professor Kihwang Lee; kie114@ajou.ac.kr and Dr Jeong Hun Kim;

steph25@snu.ac.kr

\section{ABSTRACT}

Introduction Systemic histaminergic activity is elevated in patients with diabetes mellitus. There are a few studies suggesting that histamine is implicated in the pathogenesis of diabetes, but the exact role of histamine in the development of diabetic retinopathy is unclear. The aim of this study was to investigate the role of histamine receptor $\mathrm{H} 4$ (HRH4) in the regulation of retinal pigment epithelium (RPE)-derived pro-angiogenic and antiangiogenic factors under diabetic conditions.

Research design and methods The levels of vascular endothelial growth factor (VEGF), interleukin-6 (IL-6), histamine and histidine decarboxylase (HDC) in the serum and vitreous samples of patients with diabetes were compared with those of patients without diabetes. The effect of hyperglycemia on expression levels of HRH4, VEGF, IL-6 and pigment epithelium-derived factor (PEDF) in the RPE was determined. The role of HRH4 in high glucoseinduced regulation of VEGF, IL-6 and PEDF in ARPE-19 cells and the underlying regulatory mechanism were verified using an RNA interference-mediated knockdown study. Results The serum and vitreous levels of VEGF, IL-6, histamine and HDC were more increased in patients with diabetic retinopathy than in patients without diabetes. HRH4 was overexpressed in RPE both in vitro and in vivo. Histamine treatment upregulated VEGF and IL-6 and downregulated PEDF expression in ARPE-19 cells cultivated under hyperglycemic conditions. Hyperglycemiainduced phosphorylation of p38 and subsequent upregulation of VEGF and IL-6 and downregulation of PEDF were dampened by small interfering RNA-mediated knockdown of HRH4 in ARPE-19 cells.

Conclusions Taken together, $\mathrm{HRH} 4$ was a critical regulator of VEGF, IL-6 and PEDF in the RPE under hyperglycemic conditions and the p38 mitogen-activated protein kinase pathway mediated this regulatory mechanism.

\section{INTRODUCTION}

Diabetic retinopathy is a sight-threatening microvascular complication of diabetes mellitus, the incidence of which is as high as $3.4 \%$ in the USA. ${ }^{1}$ Clinically, pathological

\section{Significance of this study}

What is already known about this subject?

- The retinal pigment epithelium (RPE) is an important cellular source of pro-angiogenic and antiangiogenic mediators.

- Histamine is suggested to be involved in the pathogenesis of diabetic microvascular complications including diabetic retinopathy.

- Histamine receptor $\mathrm{H} 4$ is expressed in the centra nervous system.

What are the new findings?

- The vitreous concentrations of histamine and histidine decarboxylase were increased in patients with diabetic retinopathy.

- The expression of histamine receptor $\mathrm{H} 4$ is increased in the RPE of mice with diabetes.

- Under diabetic condition, the activation of histamine/ histamine receptor $\mathrm{H} 4$ axis results in an imbalance of RPE-derived pro-angiogenic and anti-angiogenic factors via $\mathrm{p} 38$ signaling pathway.

How might these results change the focus of research or clinical practice?

> Our results suggest that histamine/histamine receptor $\mathrm{H} 4$ axis is activated in the eyes with diabetic retinopathy and histamine receptor $\mathrm{H} 4$ could be a potential therapeutic target in diabetic retinopathy.

retinal neovascularization and diabetic macular edema are two leading causes of vision loss in patients with diabetic retinopathy. Vascular endothelial growth factor (VEGF), a dominant pro-angiogenic and vasopermeable factor, is a key pathogenic molecule in the development of these complications. Thus, anti-VEGF agent monotherapy is currently used as a standard therapeutic measure in patients 
with proliferative diabetic retinopathy ${ }^{2}$ and diabetic macular edema. ${ }^{34}$ However, due to several limitations of anti-VEGF monotherapy, novel therapeutic targets in diabetic retinopathy are required for patients with diabetic retinopathy.

Histamine is a representative inflammatory mediator, which strongly induces vascular hyperpermeability. Several studies have suggested a relationship between diabetes and histamine. Since the pioneering work of Gill et al, which revealed that plasma histamine concentration is elevated in patients with diabetes, ${ }^{5}$ the activation of the histaminergic system has been repeatedly reported by diverse in vivo studies involving experimental animal model of diabetes. ${ }^{67}$ In terms of diabetic retinopathy, histamine synthesis is increased in the retina of rats with diabetes. ${ }^{8}$ Moreover, according to a pilot study, dual inhibition of $\mathrm{H} 1$ and $\mathrm{H} 2$ receptors prevents the breakdown of the blood retinal barrier (BRB) in patients with nonproliferative diabetic retinopathy. ${ }^{9}$ Although there are indirect data suggesting that histamine is implicated in the pathogenesis of diabetic retinopathy, the exact pathogenic role of histamine in the development of diabetic retinal complications remains to be elucidated. The biological activity of histamine is mediated by histamine receptors; to date, four subtypes of histamine receptors $(\mathrm{H} 1-\mathrm{H} 4)$ have been cloned. In the central nervous system (CNS), $\mathrm{H} 1$ and $\mathrm{H} 2$ are the two major subtypes of histamine receptors. According to recent studies, $\mathrm{H} 4$ receptor is also expressed in the CNS, mainly in vascular endothelial cells ${ }^{10}$ and the choroidal plexus epithelium. ${ }^{11}$

As a neuroepithelium, the retinal pigment epithelium (RPE) has much in common with the choroidal plexus epithelium. Physiologically, the RPE expresses diverse secretory factors to maintain retinal homeostasis. For example, the pigment epithelium-derived factor (PEDF) secreted from RPE suppresses abnormal chorioretinal neovascularization, ${ }^{12} 13$ and VEGF from RPE supports the survival of photoreceptors and the maintenance of the choriocapillaris. ${ }^{14}$ Under diabetic conditions, the physiological balance between RPEderived pro-angiogenic and anti-angiogenic factors is impaired. The production of pro-angiogenic factors such as VEGF ${ }^{15}$ and interleukin-6 (IL-6) ${ }^{16}$ are increased and the secretion of PEDF, an anti-angiogenic peptide, is decreased ${ }^{17}$ in RPE.

In this study, the intraocular histaminergic activity of patients with and without diabetes was compared. Furthermore, the expression of $\mathrm{H} 4$ receptor was determined in the retina of streptozotocin-induced mice wth diabetes and the role of $\mathrm{H} 4$ receptor in regulating RPE-driven pro-angiogenic and antiangiogenic molecules was investigated. Finally, the underlying mechanism of H4-mediated regulation of RPE-driven pro-angiogenic and antiangiogenic molecules was explored in the diabetic microenvironment.

\section{RESEARCH DESIGN AND METHODS}

\section{Study participants}

Patients who underwent pars plana vitrectomy in the Ajou University Hospital were enrolled into the study. Overall, the vitreous of 20 eyes from 20 patients with diabetes, and 20 eyes from 20 patients without diabetes were included. Demographic data, including age, sex, causes of vitrectomy, duration of diabetes mellitus and types of diabetic retinopathy were collected.

\section{Materials}

Mannitol, D-glucose and histamine were purchased from Sigma (St. Louis, Missouri, USA). The extracellular signal-regulated kinase (ERK) inhibitor, U0126, the c-Jun N-terminal kinase (JNK) inhibitor, SP600125 and the p38 mitogen-activated protein kinase (p38 MAPK) inhibitor, SB203580 were obtained from Cell Signaling Technology (Danvers, Massachusetts, USA). Antibodies to the histamine receptor H4 (HRH4) were purchased from Abcam (Cambridge, Massachusetts, USA). Antibodies against p-ERK, ERK, p-JNK, JNK, p-p38 and p38 were obtained from Cell Signaling Technology. $\beta$-Actin antibody was purchased from Santa Cruz Biotechnology (Santa Cruz, California, USA). IRDYE 800CW goat antimouse or antirabbit secondary antibodies were from LI-COR (Lincoln, Nebraska, USA). VEGF and IL-6 ELISA kits were obtained from R\&D Systems (Minneapolis, Minnesota, USA). The histamine ELISA kit was purchased from Enzo Life Sciences (Lausen, Switzerland). The histidine decarboxylase (HDC) ELISA kit was obtained from MyBioSource (San Diego, California, USA). All chemicals and buffers used in the study were purchased from Sigma.

\section{Preparation of human serum and the vitreous}

Blood samples and vitreous specimens were collected from patients with and without diabetic retinopathy. Blood samples were centrifuged at $3000 \mathrm{rpm}$ for $10 \mathrm{~min}$ at $4^{\circ} \mathrm{C}$ and stored at $-70^{\circ} \mathrm{C}$. Vitreous specimens were obtained using the standard dry sampling technique, as follows: under either general or local anesthesia, vitreous aspiration was performed via a single-port pars plana approach. Initial undiluted vitreous specimen (dry tap, $0.5 \mathrm{~mL}$ ) was centrifuged at $3000 \mathrm{rpm}$ for $10 \mathrm{~min}$ at $4^{\circ} \mathrm{C}$ and then stored at $-70^{\circ} \mathrm{C}$ until subsequent analysis.

\section{Animal model of diabetic retinopathy}

All experimental procedures involving animals were performed based on relevance to the Association for Research in Vision and Ophthalmology statement for use of animals in ophthalmic and vision research. Male C57BL $/ 6, d b / m$ and $d b / d b$ mice aged 6 weeks were purchased from the Central Animal Laboratory and maintained in a specific pathogen-free facility at Seoul National University. To establish the mouse model of type 1 diabetes, streptozotocin (200 mg/ 
$\mathrm{kg}$ ) dissolved in $0.1 \mathrm{M}$ sodium citrate buffer ( $\mathrm{pH} 4.5$ ) was injected into the peritoneal cavity of $\mathrm{C} 57 \mathrm{BL} / 6$ mice. For control C57BL/6 mice, a sham injection ( $0.1 \mathrm{M}$ sodium citrate buffer) was performed in the same manner. Blood glucose levels were determined 3 days after injection, and hyperglycemia was defined as whole blood glucose levels $>300 \mathrm{mg} / \mathrm{dL}$. C57BL/6 mice (either control or mice with type 1 diabetes) were euthanized 13 weeks after injection, while $\mathrm{db} / \mathrm{m}$ and $d b / d b$ mice were euthanized at 25 weeks of age. For RPE flat mounts, mouse eyes were immediately fixed in $4 \%$ paraformaldehyde in $0.1 \mathrm{M}$ phosphate buffer for $10 \mathrm{~min}$ at room temperature, and then transferred into phosphate-buffered saline (PBS). The cornea and lens were removed, and the retina was carefully peeled off. The remaining eyecups contained the RPE and choroid. The eyecups were dissected into quarters by four radial cuts from the periphery toward the optic disc, and then blocked in $0.25 \%$ Triton X-100 in Tris-buffered saline (TBST) with 10\% fetal bovine serum (FBS, Invitrogen, FBS002) at room temperature for 1 hour. The RPE/ choroid flat mounts were incubated with a primary antibody in TBST containing 3\% FBS and $1 \%$ bovine serum albumin (BSA) at $4^{\circ} \mathrm{C}$ overnight with gentle shaking. After washing with TBS for $10 \mathrm{~min}$, three times at room temperature, the flat mounts were incubated with the appropriate secondary antibody in TBST containing 1\% BSA for $30 \mathrm{~min}$ at room temperature, followed by washing with TBS for $10 \mathrm{~min}$, three times. Nuclei were stained with $4^{\prime}, 6$ diamidino-2'-phenylindole dihydrochloride (Invitrogen; D8417) for $10 \mathrm{~min}$ at room temperature. The flat mounts were washed with TBS, mounted in VECTASHIELD mounting medium (Vector Laboratories, H1000; Burlingame, California, USA), and images were acquired using a Leica TCS SP8 confocal microscope. The primary antibody was antimouse HRH4 (1:200; Biorbyt, orb312266; Cambridge, UK). The secondary antibody was antirabbit IgG conjugated to Alexa Fluor 488 (1:500; Invitrogen).

\section{Cell culture and treatment}

ARPE-19 cells, a cell-line derived from human RPE, were obtained from the American Type Culture Collection (Manassas, Virginia, USA). The cells were cultured in Dulbecco's modified Eagle's medium (Welgene, Deagu, Korea) containing 10\% heat-inactivated FBS and 100 U/ $\mathrm{mL}$ penicillin/streptomycin (Gibco, New York, USA). Cultures were maintained in a humidified incubator at $37^{\circ} \mathrm{C}$ with $5 \% \mathrm{CO}_{2}$. For these experiments, FBS was completely removed. The requirement for osmotically controlled conditions was accomplished by the addition of $25 \mathrm{mM}$ mannitol for 48 hours, and the requirement for high glucose was accomplished by the addition of $25 \mathrm{mM}$ D-glucose for 48 hours. After that, $0.1 \mathrm{mM}$ histamine was added to cells for 8 hours.
Determination of VEGF, IL-6, histamine and HDC levels by ELISA

The levels of VEGF, IL-6, histamine and HDC in the serum, vitreous and cell supernatants were detected using commercial ELISA kits (R\&D Systems, ENZO, MyBioSource), according to the manufacturers' protocol.

\section{Western blot analysis}

Cells were lysed using radioimmunoprecipitation assay buffer with protease inhibitors. Protein concentration was determined using a DC protein assay kit (Bio-Rad) according to the manufacturer's protocols. Samples (50 $\mu \mathrm{g})$ were mixed with the proper amount of $4 \mathrm{X}$ sample buffer, analyzed by $4 \%-20 \%$ sodium dodecyl sulfate polyacrylamide gel electrophoresis (Bio-Rad), and transferred onto polyvinylidene fluoride membranes. Membranes were incubated with primary and secondary antibodies in PBS containing $0.05 \%$ Tween-20 and were then washed three times. After the washes, the immunoblots were developed using the Odyssey Infrared Imaging System (LI-COR Biosciences). The band intensity was analyzed using ImageJ software.

\section{Real-time PCR}

For messenger RNA (mRNA) analysis, total RNA was extracted from cultured cells using the TRIzol reagent, according to the manufacturer's protocols (Invitrogen). complementary DNA (cDNA) was prepared using a cDNA synthesis kit (Takara) according to the manufacturer's instructions. Then, the synthesized cDNA was amplified using the SYBR Green I Kit (Takara). PCR was performed using the ABI PRISM $7900 \mathrm{HT}$ sequence detection system. Cycling conditions were as follows: $95^{\circ} \mathrm{C}$ for $30 \mathrm{~s}, 95^{\circ} \mathrm{C}$ for $5 \mathrm{~s}$ and $60^{\circ} \mathrm{C}$ for $1 \mathrm{~min}$ for 40 cycles. All data were normalised to those of glyceraldehyde 3-phosphate dehydrogenase, and mRNA expression levels were calculated using the delta-delta $\mathrm{Ct}$ method $\left(2^{-\Delta \Delta \mathrm{Ct}}\right)$. The primer sequences used are listed in online supplemental table 1.

\section{Small interfering RNA transfection}

Control small interfering RNA (siRNA) and the siRNA targeting HRH4 were purchased from Santa Cruz Biotechnology. To knockdown HRH4, cells were transiently transfected with siRNAs for 24 hours at a final concentration of $40 \mathrm{nM}$ using Lipofectamine 2000 (Invitrogen), according to the manufacturer's protocols.

\section{Statistical analysis}

All experiments were performed at least three times and each result is reported as the mean \pm SEM. When comparing two groups, the Student's t-test was used for parametric tests and the Mann-Whitney $\mathrm{U}$ test was used for non-parametric tests. A $p$ value of $<0.05$ was considered to be statistically significant. 
Table 1 Baseline characteristics of patients without diabetes and with diabetes

\begin{tabular}{llll}
\hline Variables & Control & DR without VH & DR with VH \\
\hline No. of eyes/atients & $20 / 20$ & $8 / 8$ & $12 / 12$ \\
\hline Men/Women (patients) & $5 / 15$ & $2 / 6$ & $7 / 12$ \\
\hline Age (years) & $59.3 \pm 17.6$ & $62.3 \pm 9.9$ & $48.17 \pm 10.5$ \\
\hline Causes of operation (eyes) & & & \\
\hline ERM & 9 & 4 & 0 \\
\hline MH & 4 & 0 & 0 \\
\hline VMTS & 2 & 2 & 0 \\
\hline RRD & 5 & 0 & 0 \\
\hline Vitreous hemorrhage & 0 & 0 & 11 \\
\hline TRD & 0 & 0 & 1 \\
\hline ME & 0 & 2 & 0 \\
\hline Diabetes duration (years) & & $8.9 \pm 4.7$ & $13.2 \pm 9.3$ \\
\hline Vitreous hemorrhage (eyes) & 0 & 0 & 12 \\
\hline
\end{tabular}

Data are presented as the mean \pm SD.

$\mathrm{DR}$, diabetic retinopathy; ERM, epiretinal membrane; $\mathrm{ME}$, macular edema; $\mathrm{MH}$, macular hole; RRD, rhegmatogenous retinal detachment; TRD, tractional retinal detachment; $\mathrm{VH}$, vitreous hemorrhage; VMTS, vitreomacular traction syndrome.

\section{RESULTS}

\section{Levels of VEGF, IL-6, histamine and HDC in the serum and} vitreous of patients with diabetes mellitus

To evaluate the effect of diabetes on the levels of VEGF, IL-6, histamine and HDC, serum and vitreous fluid was obtained from patients with $(\mathrm{n}=20)$ and without diabetic retinopathy $(n=20)$ who underwent pars plana vitrectomy. Among the diabetic eyes, 11 had vitreous hemorrhage, while the other 9 did not. The clinical characteristics of patients are presented in table 1. Serum levels of VEGF, IL-6, histamine and HDC significantly increased to a greater extent in patients with diabetic retinopathy than in the control patients, regardless of the presence of vitreous hemorrhage (figure 1A-D). Moreover, vitreous levels of VEGF, IL-6, histamine and HDC were also significantly higher in both diabetic retinopathy groups than in the control group (figure $1 \mathrm{E}-\mathrm{H}$ ). However, in the eyes of patients with diabetes, both serum and vitreous levels of VEGF, IL-6, histamine and HDC were not significantly different, with regard to the presence of vitreous hemorrhage (figure $1 \mathrm{~A}-\mathrm{H}$ ).

\section{Overexpression of HRH4 in the RPE under diabetic conditions in vivo and in vitro}

The expression pattern of HRH4 was analyzed in the retinas of patients with diabetes, the RPE-choroid-scleral complex of controls, streptozotocin-induced C57BL/6, $d b / m$ and $d b / d b$ mice with diabetes ( $\mathrm{n}=3$ for each group of mice). The RPE of streptozotocin-induced C57BL/6 mice with diabetes showed strong HRH4 expression, especially in the plasma membrane, while the RPE of sham-injected control mice demonstrated very weak HRH4 expression, in a scattered pattern. HRH4 expression level in the RPE of $d b / d b$ mice was higher compared with that of age-controlled $d b / \mathrm{m}$ mice (figure 1I). As HRH4 is highly expressed in the RPE of mice wth diabetes, the effect of glucose concentration on the expression of HRH4 in RPE was tested in vitro. ARPE-19 cells were treated with mannitol $(25 \mathrm{mM})$ or high glucose $(25 \mathrm{mM})$ containing medium for 48 hours. HRH4 expression level was higher in ARPE-19 exposed to high glucose compared with that in the osmotically controlled group (figure $1 \mathrm{~J}$ ). In other words, HRH4 was overexpressed in the exposed ARPE-19 cells. To verify whether the expression of this receptor was regulated at the level of transcription, levels of $\mathrm{HRH} 4$ mRNA in ARPE-19 cells cultivated under hyperglycemic or hyperosmotic conditions were quantified by quantitative real-time PCR. The level of HRH4 transcript was significantly higher in the high glucose group than in the hyperosmotic group (figure 1K).

\section{Role of the histamine/HRH4 axis in the regulation of VEGF, IL-6 and PEDF expression in ARPE-19 cells exposed to high glucose}

As previously noted, VEGF and IL-6 levels were higher in ARPE-19 cells cultivated in high glucose medium than in cells grown in osmotically controlled medium (5.22 \pm 0.28 -fold and 3.45 \pm 0.45 -fold, respectively). Histamine supplementation potentiated the high glucose-induced transcriptional upregulation of VEGF (from 5.22 \pm 0.28 -fold to 9.78 \pm 1.61 -fold) and IL-6 (from $3.45 \pm 0.45$-fold to $5.98 \pm 1.05$-fold) in ARPE-19 cells (figure 2A,B). In contrast, PEDF expression was downregulated in ARPE-19 cells exposed to high glucose medium, and the fold change of PEDF expression between the high glucose and osmotically controlled groups increased on histamine supplementation (figure 2C).

Next, to determine the role of HRH4 in the histamineinduced exaggeration of high glucose-mediated 

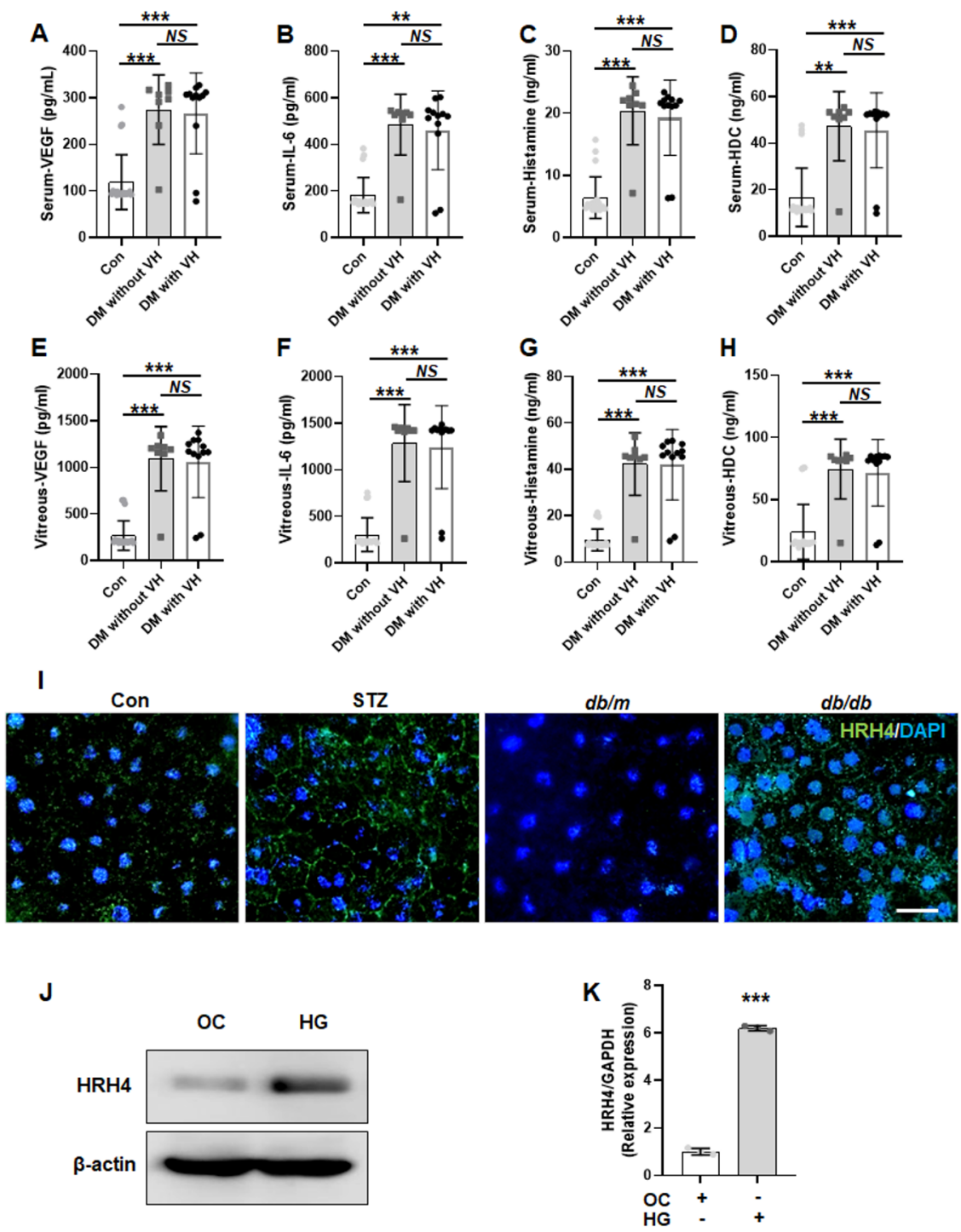

Figure 1 Increased histaminergic activity in the eyes of patients with diabetic retinopathy and upregulation of $\mathrm{HRH} 4$ expression in the retinal pigment epithelium of experimental diabetic conditions. (A-H) Levels of VEGF, IL-6, histamine and HDC in the serum and vitreous of patients with diabetic retinopathy ( 9 without $\mathrm{VH}$ and 11 with $\mathrm{VH}$ ) are shown, as determined using ELISA and compared with those of non-diabetic controls $(n=20)$. (I) Representative images of the RPE/choroid/scleral complex flat mounts from sham or streptozotocin-injected C57BL/6 mice (13 weeks after injection), db/m and db/db mice (25 weeks old) immunostained using the HRH4 antibody. Scale bar, $25 \mu \mathrm{m}$. (J, K) Protein and messenger RNA (mRNA) transcript levels of HRH4 in ARPE-19 cells cultivated in high glucose medium (D-glucose, $25 \mathrm{mM}$ ) or under osmotically controlled medium (mannitol, $25 \mathrm{mM}$ ) are shown, as determined using immunoblot assay and quantitative real-ime $\mathrm{PCR}$, respectively. ${ }^{* *} \mathrm{P}<0.01$, ${ }^{* * *} \mathrm{p}<0.001$. Error bars represent the mean \pm SEM. Con, control; DAPI, 4',6-diamidino-2-phenylindole; DM, diabetes mellitus; HDC, histidine decarboxylase; HG, high glucose; HRH4, histamine receptor H4; IL-6, interleukin 6; NS, not significant; OC, osmotically controlled; STZ, streptozotocin-induced mice wth diabetes; VEGF, vascular endothelial growth factor; VH, vitreous hemorrhage.

upregulation of VEGF and IL-6 and the downregulation of PEDF in ARPE-19 cells, HRH4 was silenced in ARPE-19 cells using siRNA. In the histamine-treated ARPE-19 cells, expression of VEGF and IL-6 was significantly suppressed by siRNA-mediated knockdown of HRH4 (figure 2D,E). Notably, HRH4 knockdown inhibited the histamine-induced transcription of VEGF and IL-6, both in the high glucose group and the osmotically controlled group. In addition, the downregulated PEDF expression in ARPE-19 cells cultivated under high glucose conditions was ameliorated by $H R H 4$ silencing (figure $2 \mathrm{~F}$ ).
To confirm the role of HRH4 in regulating histaminemediated VEGF and IL-6 secretion in ARPE-19 cells, an ELISA was performed to determine levels of VEGF and IL-6 in ARPE-19 conditioned medium. The protein levels of VEGF and IL- 6 were higher in the conditioned medium of ARPE-19 which cultivated in high glucose concentration than that of osmotically controlled group. Silencing HRH4 significantly reduced the concentration of VEGF and IL-6 in the ARPE-19 conditioned medium, regardless of the glucose concentration of the culture medium (figure $2 \mathrm{G}, \mathrm{H}$ ). These data indicated that HRH4 overexpression induced by high glucose-mediated histamine-induced upregulation of 


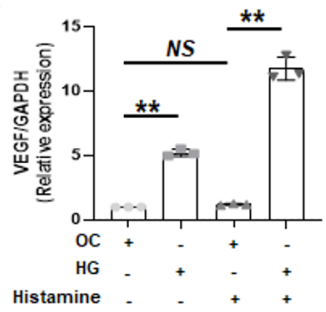

B

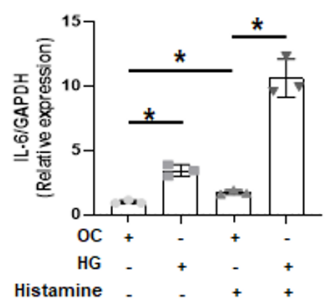

C

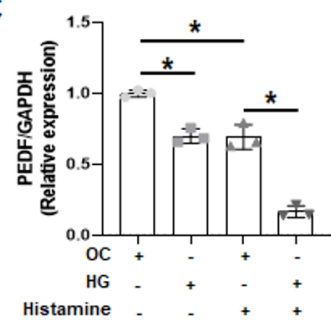

D

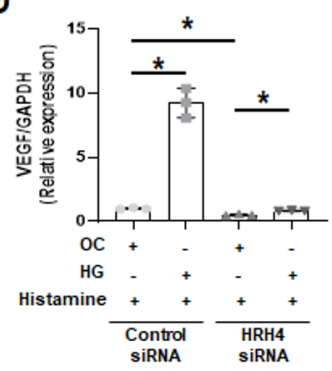

E

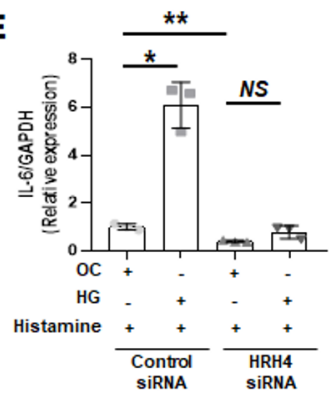

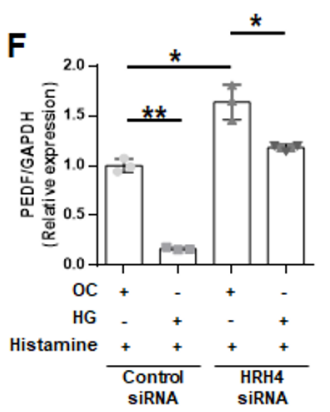

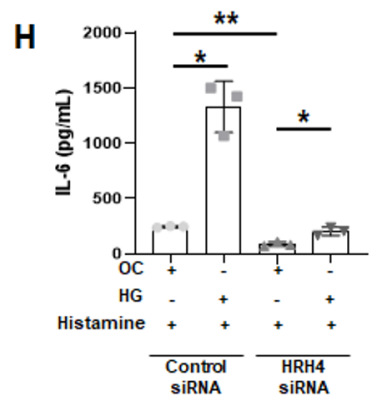

Figure 2 Role of HRH4 in modulating the expression of pro-angiogenic and anti-angiogenic factors in ARPE-19 cells. ARPE19 cells were cultivated in high glucose ( $25 \mathrm{mM}$ D-glucose) or osmotically controlled ( $25 \mathrm{mM}$ mannitol) medium for 48 hours and then treated with or without histamine $(0.1 \mathrm{mM})$. (A-C) Messenger RNA (mRNA) levels of VEGF, IL-6 and PEDF are shown, as determined 8 hours after histamine treatment. ARPE-19 cells transfected with $40 \mathrm{nM}$ negative control small interfering RNA (siRNA) or HRH4 siRNA were cultivated with high-dose D-glucose-containing $(25 \mathrm{mM})$ or mannitol-containing $(25 \mathrm{mM})$ medium. After 48 hours, ARPE-19 cells were treated with or without histamine $(0.1 \mathrm{mM})$ for 8 hours. (D-F) mRNA levels of VEGF, IL-6 and PEDF are shown, as measured by quantitative real-time PCR. (G, H) Levels of VEGF and IL-6 in ARPE-19 cell-conditioned medium are shown, as determined using an ELISA. All experiments are performed in triplicate, and error bars represent the mean \pm SEM. ${ }^{\star} P<0.05,{ }^{* *} p<0.01$. GAPDH; glyceraldehyde 3 -phosphate dehydrogenase, $\mathrm{HG}$, high glucose; HRH4, histamine receptor H4; IL-6, interleukin 6; NS, not significant; OC, osmotically controlled; PEDF, pigment epithelium-derived factor; VEGF, vascular endothelial growth factor.

VEGF and IL-6 and downregulation of PEDF expression in ARPE-19 cells.

\section{Activation of HRH4 and the downstream p38 MAPK for histamine-induced regulation of VEGF, IL-6 and PEDF expression in ARPE-19 cells}

Several signaling pathways involving MAPKs such as ERK, JNK and p38 are activated by high glucose conditions in a diverse cellular context; these MAPKs are involved in the regulation of VEGF and IL-6 under diabetic conditions. Thus, the role of these kinases in the upregulation of HRH4 and HRH4-mediated overexpression of VEGF and IL-6 was investigated. First, it was verified whether high glucose induced the activation of ERK, JNK and p38 in ARPE-19 cells and whether inhibition of these MAPKs affected levels of HRH4 in ARPE-19 cells by western blot analysis. As shown in figure 3A-C, the phosphorylation of ERK, JNK and p38 was significantly increased in high glucose-treated cells. The phosphorylation of each MAPK was effectively suppressed by specific inhibitors of ERK (U0126), JNK (SP600125) and p38 (SB203580), but HRH4 expression was not changed by MAPK inhibitors in high glucosetreated ARPE-19 cells. These results suggested that the ERK, JNK and p38 signaling pathways were activated by high glucose conditions, not by high glucose-induced HRH4 upregulation, in ARPE-19 cells. 
A

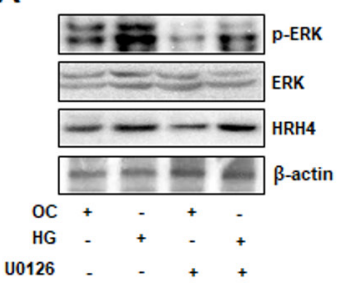

D

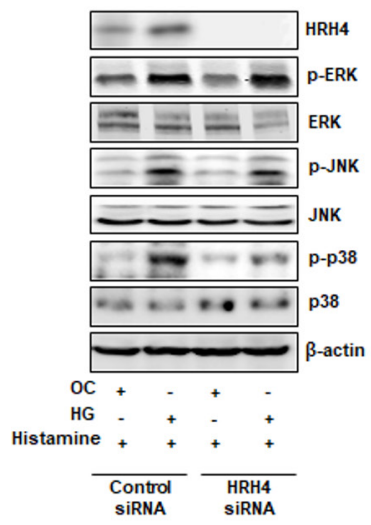

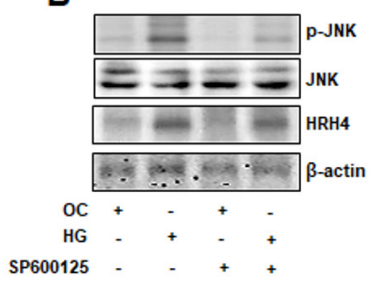

$\mathbf{E}$

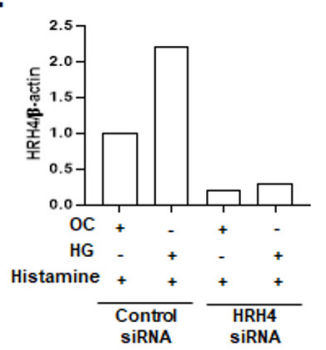

G

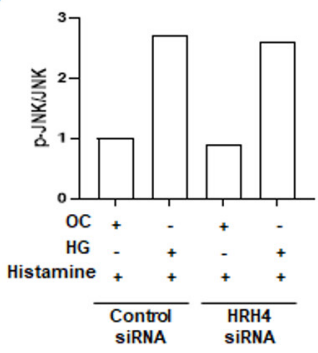

C

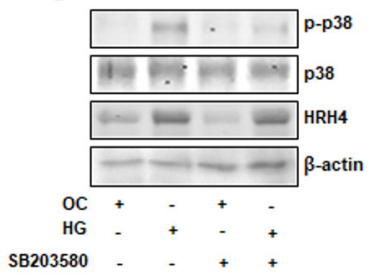

$\mathbf{F}$

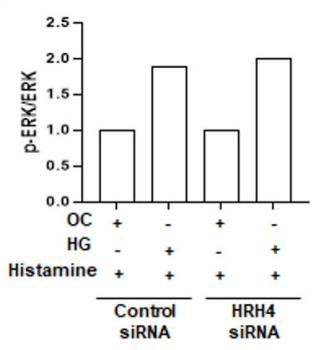

H

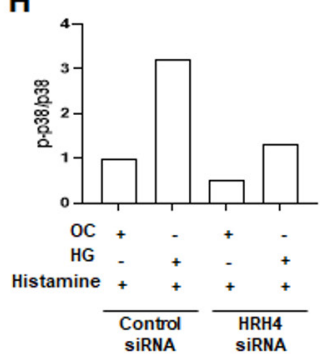

Figure 3 Regulation of the activity of the p38 mitogen-activated protein kinase signaling pathway by the histamine $\mathrm{H} 4$ receptor in ARPE-19 cells cultivated under high glucose conditions. ARPE-19 cells pretreated with selective inhibitors of ERK (U0126), JNK (SP600125) and p38 (SB203580) for $60 \mathrm{~min}$ were incubated with high glucose (25 mM D-glucose) or osmotically controlled ( $25 \mathrm{mM}$ mannitol) medium for 48 hours, and then treated with histamine ( $0.1 \mathrm{mM}$, 8 hours). (A-C) Levels of $p$-ERK, ERK, p-JNK, JNK, p-p38, p38 and HRH4 are shown, as determined using an immunoblot assay. $\beta$-Actin is used as the internal control. (D) ARPE-19 cells transfected with either scrambled small interfering RNA (siRNA) or HRH4 siRNA were cultivated with high glucose (25 mM D-glucose) or osmotically controlled ( $25 \mathrm{mM}$ mannitol) medium for 48 hours, and then treated with histamine (0.1 mM) for 8 hours. Levels of p-ERK, ERK, p-JNK, JNK, p-p38, p38 and HRH4 are shown, as evaluated by immunoblotting. (E-H) Densitometric analysis was performed using ImageJ. ERK, extracellular signal-regulated kinase; HG, high glucose; HRH4, histamine receptor H4; JNK, C-Jun N-terminal kinase; OC, osmotically controlled.

Next, the involvement of these MAPKs in the signaling pathway downstream of HRH4 was tested. In ARPE-19 cells treated with histamine, HRH4 knockdown significantly suppressed the phosphorylation of p38, without affecting the phosphorylation of other MAPKs (figure 3D-H). In both the high glucose and osmotically controlled groups, the ratio of p-p38/p38 decreased with HRH4 knockdown. Taken together, it was postulated that HRH4 regulated p38 signaling in high glucose-treated ARPE-19 cells. Moreover, it was confirmed that histamine-induced upregulation of VEGF and IL-6 expression and histaminemediated downregulation of PEDF were blocked by the selective inhibitor against p38. However, the p38 inhibitor did not affect histamine-induced regulation of VEGF, IL-6 and PEDF expression in ARPE-19 cells transfected with HRH4 siRNA (figure 4A-C). Thus, the histamineinduced regulation of VEGF, IL-6 and PEDF expression via HRH4 in RPE was mediated by activation of p38 (figure 5).

\section{DISCUSSION}

In this study, it was first shown that vitreoretinal histaminergic activity was enhanced in patients with diabetic retinopathy. The vitreous levels of HDC and histamine, as well as those of the pro-angiogenic peptides VEGF and IL-6, were significantly increased in patients with diabetic retinopathy. Patients with diabetic retinopathy were subgrouped according to the presence of vitreous hemorrhage because blood contamination might affect the vitreous levels of these peptides. However, the presence of vitreous hemorrhage did not affect the levels of these peptides. Because vitreous sampling was performed at least several weeks after the onset of hemorrhage, it 

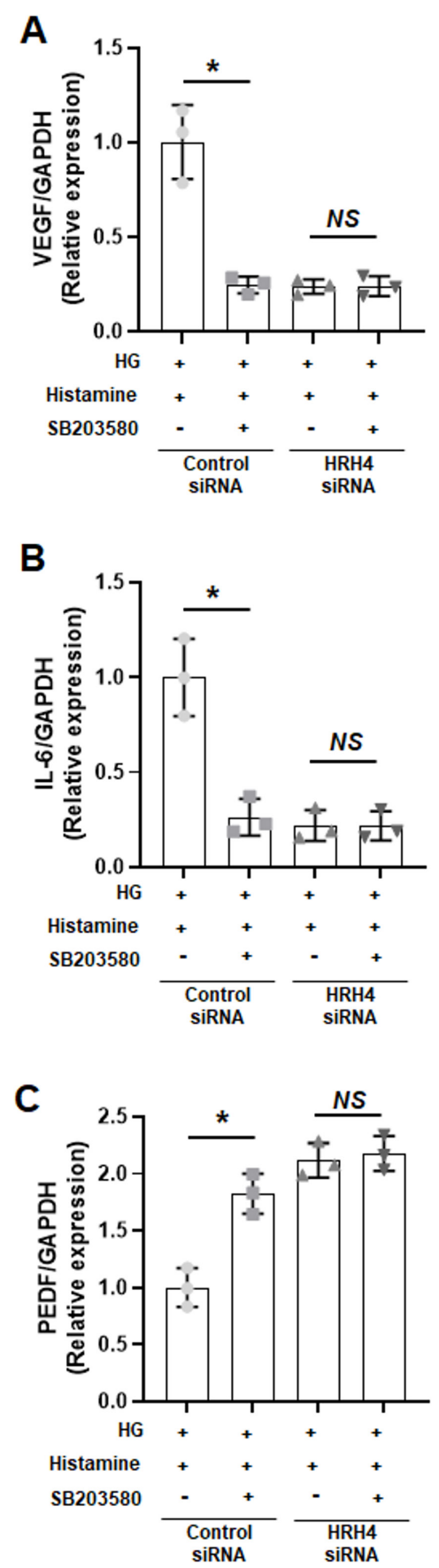

Figure 4 Role of the HRH4/p38 mitogen-activated protein kinase signaling pathway in histamine-induced regulation of VEGF, IL-6 and PEDF expression in ARPE-19 cells. ARPE-19 cells transfected either with negative control small interfering RNA (siRNA) or with HRH4 siRNA were exposed to high glucose-containing $(25 \mathrm{mM})$ medium for 48 hours. Then, ARPE-19 cells from each group were incubated with histamine-containing $(0.1 \mathrm{mM})$ medium, in the presence and absence of SB203580, a specific inhibitor of p38. (A-C) Levels of VEGF, IL-6 and PEDF messenger RNA (mRNA) are shown, as determined by quantitative real-time PCR. All experiments are performed in triplicate, and error bars represent the mean \pm SEM. ${ }^{*} P<0.05$. GAPDH; glyceraldehyde 3-phosphate dehydrogenase, $\mathrm{HG}$, high glucose; $\mathrm{HRH}$, histamine receptor $\mathrm{H} 4$; IL-6, interleukin 6; NS, not significant; PEDF, pigment epithelium-derived factor; VEGF, vascular endothelial growth factor. was postulated that these blood peptides were decomposed in the vitreous cavity at the time of sampling.

HRH4 was overexpressed in the RPE of mice with both type 1 and type 2 diabetes. RPE is a cellular component of the outer BRB. Because breakdown of both the inner and outer BRB contributes to the pathogenesis of diabetic retinopathy, it is believed that the RPE exerts only a passive role in the development of diabetic retinopathy. However, in recent years, the RPE has been identified as an active cellular component which contributes to the progression of diabetic retinopathy. ${ }^{18}$ In diabetic retinopathy, RPE-secreted peptides are easily dispersed into the neural retina because the outer BRB is dysfunctional. ${ }^{19}$

Retinal neovascularization and macular edema are two major pathological events that occurs during the progression of diabetic retinopathy. Pathological angiogenesis is the result of imbalance between pro-angiogenic and anti-angiogenic factors. VEGF and IL-6 are representative pro-angiogenic peptides which exert a central role in the development of pathological retinal angiogenesis in diabetic retinopathy. These factors also weaken the blood-retinal barrier to induce diabetic macular edema. PEDF originating from RPE is, conversely, one of the most potent anti-angiogenic peptide expressed in the retina. Along with Müller cells, RPE has been recently identified as an important cellular source of secretory pro-angiogenic and anti-angiogenic factors under diabetic conditions. ${ }^{1516}$ However, the exact mechanism involved in the regulation of pro-angiogenic and antiangiogenic peptides in RPE under diabetic conditions remains to be elucidated. The pro-angiogenic effect of histamine has been observed in diverse pathological situations. ${ }^{20-22}$ However, the underlying cellular mechanisms of histamine-induced angiogenesis are different between the disease conditions. Furthermore, in diabetic retinas, it is not understood how histamine promotes angiogenesis. Thus, this study evaluated the role of the histamine/ HRH4 axis in modulating VEGF, IL-6 and PEDF expression in diabetic retinas.

We have shown that high glucose condition induces the upregulation of VEGF and IL-6 in RPE and histamine treatment leads to an exaggerated upregulation of VEGF and IL-6 in RPE exposed to high glucose concentration. In contrast, PEDF expression was downregulated in ARPE-19 cells under high glucose conditions and high glucose-induced PEDF downregulation was augmented by histamine supplementation. In the presence of histamine, the expression of VEGF and IL-6 was significantly downregulated by knockdown of HRH4 in ARPE-19 cells. In addition, high glucose-induced decreases in PEDF expression levels were alleviated by HRH4 knockdown in ARPE-19 cells. Interestingly, this inhibitory effect was observed in high-glucose group, and in the osmotic control group. Because basal expression of HRH4 was identified in this study, it was postulated that the histamine/HRH4 axis itself was an important regulator of VEGF, IL-6 and PEDF expression. In the diabetic retina, increased intraocular 

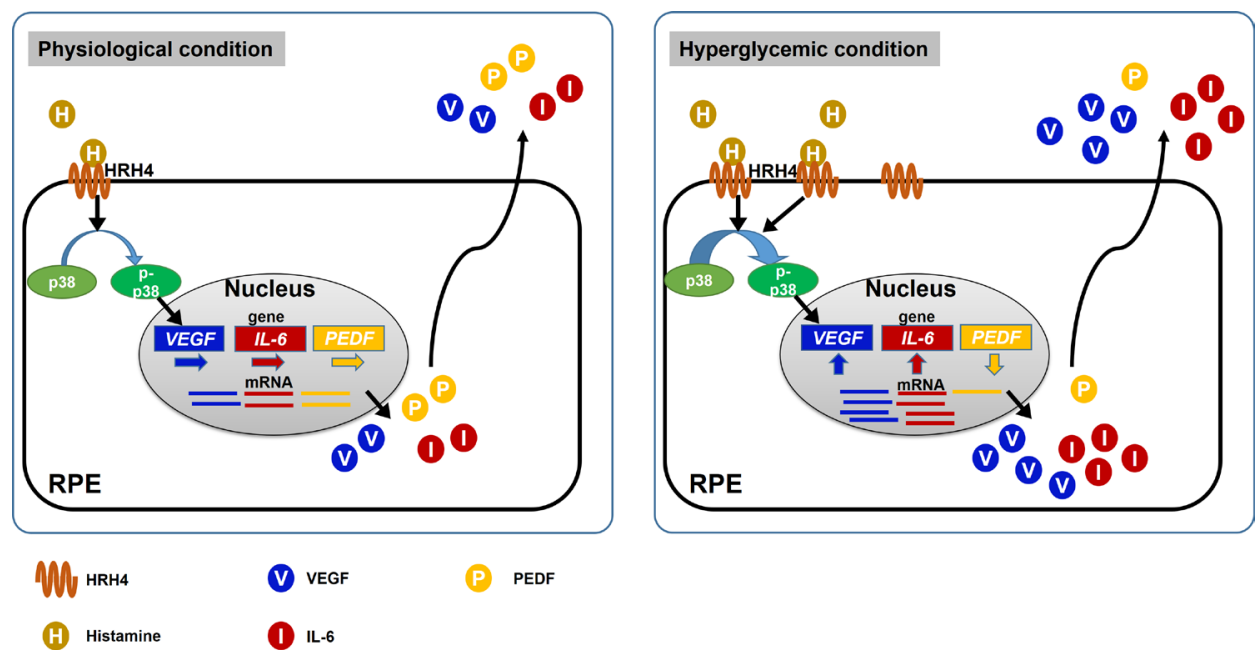

Figure 5 Schematic illustration showing the regulatory mechanism for pro-angiogenic and anti-angiogenic factor expression in the RPE of diabetic retina via H4 receptor/p38 MAPK axis. HRH4, histamine receptor H4; IL-6, interleukin-6; mRNA, messenger RNA; p38 MAPK, p38 mitogen-activated protein kinase; PEDF, pigment epithelium-derived factor; RPE, retinal pigment epithelium; VEGF, vascular endothelial growth factor.

histamine activated overexpression of HRH4 in the RPE to significantly upregulate VEGF and IL-6 and downregulate PEDF expression.

HRH4 is a $\mathrm{G}$ protein coupled receptor which differentially mediates the activation of diverse MAPKs, according to given cellular contexts. ${ }^{23}{ }^{24}$ Under diabetic conditions, MAPKs such as ERK, JNK and p38 are commonly activated in diverse cellular components, and these kinases are involved in the regulation of $\mathrm{VEGF}^{25} 26$ and IL-6 ${ }^{27}$ expression. ERK, JNK and p38 were also activated in RPE cells cultivated under high glucose conditions. Furthermore, it was shown that p38 was a downstream component of the histamine/HRH4 signaling axis. In contrast, the phosphorylation of ERK and JNK was not changed by histamine/HRH4 signaling. HRH4-induced VEGF and IL-6 overexpression and PEDF downregulation in the RPE were mediated by p38 activity, as shown using a specific inhibitor of $\mathrm{p} 38$. The activation of p38 kinase has been observed in diverse diabetic microvascular complications such as diabetic nephropathy ${ }^{28} 29$ and retinopathy. ${ }^{30}$ Moreover, several investigators have shown that the suppression of p38 leads to the improvement of diabetic retinopathy. ${ }^{31}{ }^{32}$ The current results are in concordance with these previous studies.

In conclusion, these results showed that vitreoretinal histaminergic tone was elevated in patients with diabetes and HRH4 was overexpressed in the RPE of diabetic retinas. These data suggested that HRH4 is a key regulator of histamine-induced upregulation of proangiogenic factors (VEGF and IL-6) and downregulation of the anti-angiogenic factor PEDF in the RPE. In addition, p38 mediated the histamine/HRH4-induced regulation of these pro-angiogenic and anti-angiogenic factors in the RPE of diabetic retinas. Thus, HRH4 could be a potential therapeutic target in patients with diabetic retinal complications.

\section{Author affiliations}

${ }^{1}$ Department of Ophthalmology, Asan Medical Center, University of Ulsan College of Medicine, Seoul, Republic of Korea

${ }^{2}$ Fight against Angiogenesis-Related Blindness (FARB) Laboratory, Clinical Research Institute, Seoul National University Hospital, Seoul, Republic of Korea

${ }^{3}$ Department of Biomedical Sciences, Seoul National University, Seoul, Republic of Korea

${ }^{4}$ Institute of Medical Science, Ajou University School of Medicine and Graduate School of Medicine, Suwon, Gyeonggi-do, Republic of Korea

${ }^{5}$ Department of Ophthalmology, Korea University College of Medicine, Seoul, Republic of Korea

${ }^{6}$ Youth Bio Global, Ltd, Seoul, Republic of Korea

${ }^{7}$ Biomedical Center for Animal Resource Development and Institute for

Experimental Animals, Seoul National University College of Medicine, Seoul, Republic of Korea

${ }^{8}$ Macrophage Lab, Department of Microbiology and Immunology, and Institute of Endemic Disease, Seoul National University College of Medicine, Seoul, Republic of Korea

${ }^{9}$ Department of Microbiology and Immunology, and Institute of Endemic Disease, Seoul National University College of Medicine, Seoul, Republic of Korea

${ }^{10}$ Ophthalmology, Seoul National University College of Medicine, Seoul, Republic of Korea

${ }^{11}$ Department of Ophthalmology, Ajou University School of Medicine and Graduate School of Medicine, Suwon, Gyeonggi-do, Republic of Korea

Contributors BJL, HEB, YHK, JiHK, JeHK and KL contributed to conception and design of study. HEB, J-WK and CSC contributed to data collection. BJL and HEB contributed to data analysis and interpretation. All of the authors have participated in drafting and revising the manuscript. JeHK and KL have finally approved the manuscript.

Funding Supported by grants from the K-Bio Health R\&D Project, Ministry of Health \& Welfare, Republic of Korea (H016C0001), Basic Science Research Program through the National Research Foundation of Korea (NRF) funded by the Ministry of Education (2017R1D1A1A02018439), the Bio \& Medical Technology Development Program of the National Research Foundation funded by the Korean government, MSIP (NRF-2015M3A9E6028949 to JeHK), the Creative Materials Discovery Program through the National Research Foundation of Korea (NRF) funded by Ministry of Science and ICT (2018M3D1A1058826 to JeHK) and Development of Platform Technology for Innovative Medical Measurements funded by Korea Research Institute of Standards and Science (KRISS-2019-GP2019-0013 to JeHK).

Competing interests None declared.

Patient consent for publication Obtained. 
Ethics approval The institutional review board (IRB) of Ajou University Hospital reviewed and approved this study (IRB approval number: BMR-SMP-17-190). The Institutional Animal Care and Use Committee of Seoul National University approved the animal studies. Informed consent was obtained from participants prior to their involvement in the study.

Provenance and peer review Not commissioned; externally peer reviewed.

Data availability statement Data are available on reasonable request.

Supplemental material This content has been supplied by the author(s). It has not been vetted by BMJ Publishing Group Limited (BMJ) and may not have been peer-reviewed. Any opinions or recommendations discussed are solely those of the author(s) and are not endorsed by BMJ. BMJ disclaims all liability and responsibility arising from any reliance placed on the content. Where the content includes any translated material, BMJ does not warrant the accuracy and reliability of the translations (including but not limited to local regulations, clinical guidelines, terminology, drug names and drug dosages), and is not responsible for any error and/or omissions arising from translation and adaptation or otherwise.

Open access This is an open access article distributed in accordance with the Creative Commons Attribution Non Commercial (CC BY-NC 4.0) license, which permits others to distribute, remix, adapt, build upon this work non-commercially, and license their derivative works on different terms, provided the original work is properly cited, appropriate credit is given, any changes made indicated, and the use is non-commercial. See: http://creativecommons.org/licenses/by-nc/4.0/.

ORCID iD

Byung Joo Lee http://orcid.org/0000-0003-4210-7650

\section{REFERENCES}

1 Kempen JH, O'Colmain BJ, Leske MC, et al. The prevalence of diabetic retinopathy among adults in the United States. Arch Ophthalmol 2004;122:552-63.

2 Writing Committee for the Diabetic Retinopathy Clinical Research Network, Gross JG, Glassman AR, et al. Panretinal photocoagulation vs Intravitreous ranibizumab for proliferative diabetic retinopathy: a randomized clinical trial. JAMA 2015;314:2137-46.

3 Michaelides M, Kaines A, Hamilton RD, et al. A prospective randomized trial of intravitreal bevacizumab or laser therapy in the management of diabetic macular edema (bolt study) 12-month data: report 2. Ophthalmology 2010;117:1078-86.

4 Brown DM, Nguyen QD, Marcus DM, et al. Long-Term outcomes of ranibizumab therapy for diabetic macular edema: the 36-month results from two phase III trials: rise and ride. Ophthalmology 2013;120:2013-22.

5 Gill DS, Barradas MA, Fonseca VA, et al. Plasma histamine concentrations are elevated in patients with diabetes mellitus and peripheral vascular disease. Metabolism 1989;38:243-7.

6 Gill DS, Thompson CS, Dandona P. Increased histamine in plasma and tissues in diabetic rats. Diabetes Res 1988;7:31-4.

7 Gill DS, Thompson CS, Dandona P. Histamine synthesis and catabolism in various tissues in diabetic rats. Metabolism 1990;39:815-8.

8 Carroll WJ, Hollis TM, Gardner TW. Retinal histamine synthesis is increased in experimental diabetes. Invest Ophthalmol Vis Sci 1988;29:1201-4.

9 Gardner TW, Eller AW, Friberg TR, et al. Antihistamines reduce blood-retinal barrier permeability in type I (insulin-dependent) diabetic patients with nonproliferative retinopathy. A pilot study. Retina 1995;15:134-40.

10 Karlstedt K, Jin C, Panula P. Expression of histamine receptor genes Hrh3 and Hrh4 in rat brain endothelial cells. Br J Pharmacol 2013;170:58-66.

11 Maślińska D, Laure-Kamionowska M, Maśliński KT, et al. Morphology and immuno-distribution of the histamine $\mathrm{H} 4$ receptor and histamine--releasing factor in choroid plexus of patients with paraneoplastic cerebellar degeneration. Inflamm Res 2009;58 Suppl 1:45-6.

12 Renno RZ, Youssri Al, Michaud N, et al. Expression of pigment epithelium-derived factor in experimental choroidal neovascularization. Invest Ophthalmol Vis Sci 2002;43:1574-80.

13 Duh EJ, Yang HS, Suzuma l, et al. Pigment epithelium-derived factor suppresses ischemia-induced retinal neovascularization and VEGF-induced migration and growth. Invest Ophthalmol Vis Sci 2002;43:821-9.

14 Saint-Geniez M, Kurihara T, Sekiyama E, et al. An essential role for RPE-derived soluble VEGF in the maintenance of the choriocapillaris. Proc Natl Acad Sci U S A 2009;106:18751-6.

15 Sone H, Kawakami Y, Okuda Y, et al. Ocular vascular endothelial growth factor levels in diabetic rats are elevated before observable retinal proliferative changes. Diabetologia 1997;40:726-30.

16 Holtkamp GM, Van Rossem M, de Vos AF, et al. Polarized secretion of IL- 6 and IL- 8 by human retinal pigment epithelial cells. Clin Exp Immunol 1998;112:34-43.

17 Calado SM, Alves LS, Simão S, et al. Glut1 activity contributes to the impairment of PEDF secretion by the RPE. Mol Vis 2016;22:761-70.

18 Ponnalagu M, Subramani M, Jayadev C, et al. Retinal pigment epithelium-secretome: a diabetic retinopathy perspective. Cytokine 2017;95:126-35

19 Al-Araimi M, Pal B, Poulter JA, et al. A new recessively inherited disorder composed of foveal hypoplasia, optic nerve decussation defects and anterior segment dysgenesis maps to chromosome 16q23.3-24.1. Mol Vis 2013;19:2165-72.

20 Lu Q, Wang C, Pan R, et al. Histamine synergistically promotes bFGF-induced angiogenesis by enhancing VEGF production via $\mathrm{H} 1$ receptor. J Cell Biochem 2013;114:1009-19.

21 Qin L, Zhao D, Xu J, et al. The vascular permeabilizing factors histamine and serotonin induce angiogenesis through TR3/Nur77 and subsequently truncate it through thrombospondin-1. Blood 2013;121:2154-64

22 Hegyesi H, Tóth S, Molnár V, et al. Endogenous and exogenous histamine influences on angiogenesis related gene expression of mice mammary adenocarcinoma. Inflamm Res 2007;56 Suppl 1:S37-8.

23 Dib K, Perecko T, Jenei V, et al. The histamine $\mathrm{H} 4$ receptor is a potent inhibitor of adhesion-dependent degranulation in human neutrophils. J Leukoc Biol 2014:96:411-8.

24 Beermann S, Bernhardt G, Seifert R, et al. Histamine $\mathrm{H}(1)$ - and $\mathrm{H}(4)$ receptor signaling cooperatively regulate MAPK activation. Biochem Pharmacol 2015;98:432-9.

25 Kim SR, Lee KS, Park SJ, et al. Inhibition of p38 MAPK reduces expression of vascular endothelial growth factor in allergic airway disease. J Clin Immunol 2012;32:574-86.

26 Luo X, Gu S, Zhang Y, et al. Kinsenoside ameliorates oxidative stress-induced RPE cell apoptosis and inhibits angiogenesis via Erk p38/NF-кB/VEGF signaling. Front Pharmacol 2018;9:240.

27 Patil C, Zhu X, Rossa C, et al. P38 MAPK regulates IL-1beta induced IL-6 expression through mRNA stability in osteoblasts. Immunol Invest 2004;33:213-33.

28 Adhikary L, Chow F, Nikolic-Paterson DJ, et al. Abnormal p38 mitogen-activated protein kinase signalling in human and experimental diabetic nephropathy. Diabetologia 2004;47:1210-22.

29 Komers R, Lindsley JN, Oyama TT, et al. Renal p38 MAP kinase activity in experimental diabetes. Lab Invest 2007;87:548-58.

30 Huang C, Zhu H-J, Li H, et al. P38-Mapk pathway is activated in retinopathy of microvascular disease of STZ-induced diabetic rat model. Eur Rev Med Pharmacol Sci 2018;22:5789-96.

31 Wang S, Du S, Wu Q, et al. Decorin prevents retinal pigment epithelial barrier breakdown under diabetic conditions by suppressing p38 MAPK activation. Invest Ophthalmol Vis Sci 2015;56:2971-9.

32 Du Y, Tang J, Li G, et al. Effects of p38 MAPK inhibition on early stages of diabetic retinopathy and sensory nerve function. Invest Ophthalmol Vis Sci 2010;51:2158-64. 